\title{
Rescuing protein conformation: prospects for pharmacological therapy in cystic fibrosis
}

\author{
Marina S. Gelman ${ }^{1}$ and Ron R. Kopito ${ }^{2}$ \\ ${ }^{1}$ PPD Discovery Inc., Menlo Park, California, USA \\ ${ }^{2}$ Department of Biological Sciences, Stanford University, Stanford, California, USA \\ J. Clin. Invest. 110:1591-1597 (2002). doi:10.1172/JCI200216786.
}

Cystic fibrosis (CF), a fatal autosomal recessive genetic disease that affects over 60,000 people worldwide, is caused by mutations in CFTR (1). This gene encodes the cystic fibrosis transmembrane conductance regulator protein, which functions as a $\mathrm{Cl}^{-}$channel at the apical membranes of pulmonary epithelial cells (2). The CFTR channel is also found in certain other epithelia, such as the sweat ducts and part of the gastrointestinal tract, but lung pathology is by far the most prominent cause of clinical disease in CFTR homozygotes and compound heterozygotes. Precisely how the loss of functional, surface-expressed CFTR channels and the consequent decrease in $\mathrm{Cl}^{-}$conductance lead to CF pathogenesis is controversial (3). Still, the recognition that the majority of cases of CF are the result of a defect in biogenesis or intracellular trafficking of the protein, and that the mutant protein retains at least partial function, has stimulated an intensive search for therapeutic strategies aimed at rescuing the function of the mutant CFTR. Herein, we discuss some of the results of this search and the prospects of such a protein-repair strategy in the context of CFTR biogenesis and intracellular trafficking.

\section{CFTR biogenesis}

CFTR is a polytopic integral membrane glycoprotein composed of 1,480 amino acids (Figure 1). Biogenesis of CFTR begins with the targeting of nascent chainribosome complexes to the endoplasmic reticulum (ER) membrane, followed by translocation and integration of transmembrane domains into the lipid bilayer (4) (Figure 2a, step 1). Conformational maturation of wild-type CFTR in the ER (step 2) is an inefficient process; approximately $75 \%$ of newly synthe-

\footnotetext{
Address correspondence to: Ron R. Kopito, Department of Biological Sciences, Stanford University, Stanford, California 94305-5020, USA. Phone: (650) 723-7581; Fax: (650) 723-8475; E-mail: kopito@stanford.edu.

Conflict of interest: The authors have declared that no conflict of interest exists.

Nonstandard abbreviations used: cystic fibrosis (CF); endoplasmic reticulum (ER); trimethylamine- $N$-oxide (TMAO); 4-phenyl butyrate (4PB); 8-cyclopentenyl-1,3-dipropylxanthine (CPX); deoxyspergualin (DSG); thapsigargin (TG); doxorubicin (Dox).
}

sized CFTR molecules are degraded by cytoplasmic proteasomes shortly after synthesis (step 3). Maturation of CFTR to post-ER compartments (step 4) can be readily detected as an approximately $20-\mathrm{kDa}$ decrease in electrophoretic mobility. This decrease reflects conversion by enzymes in the Golgi apparatus of the two Asn-linked glycans in the fourth extracellular loop from immature, high-mannose forms into mature, complex oligosaccharides. Once delivered to the plasma membrane, CFTR is subject to rapid internalization to a pool of subapical vesicles (step 5) that can be recycled to the plasma membrane (step 6) or delivered to lysosomes for degradation (5) (step 8).

Over 1,200 mutations and sequence variants in the CFTR gene have been linked to CF (Cystic Fibrosis Mutation Data Base, http://www.genet.sickkids.on.ca/ $\mathrm{cftr} /)$. These mutations have been grouped into four classes (2): class I mutations abrogate the synthesis of CFTR protein (Figure 2a, step 1), class II mutants are defective in protein trafficking (steps 2 and 4), class III mutations lead to the presence of unstable or nonfunctional protein at the plasma membrane (steps 5, 6, and 8), and class IV mutations interfere with channel activation and regulation by physiological agonists (step 7). Despite this large number of CFTR disease alleles, the vast majority (>90\%) of CF patients of Northern European origin have at least one copy of a single mutant allele, $\Delta$ F508, which encodes a CFTR molecule lacking a phenylalanine at position $508(1,6)$ (Figure 1).

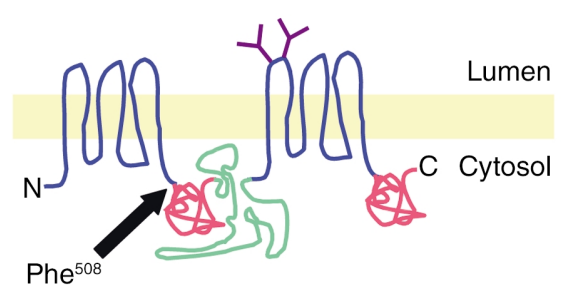

Figure 1

Cartoon representation of CFTR structure. Indicated are the transmembrane domains (blue), the two Asn-linked glycans (purple), the "R" domain (green), and the two nucleotide-binding domains (red). $\mathrm{N}$, amino terminus; $\mathrm{C}$, carboxy terminus. 

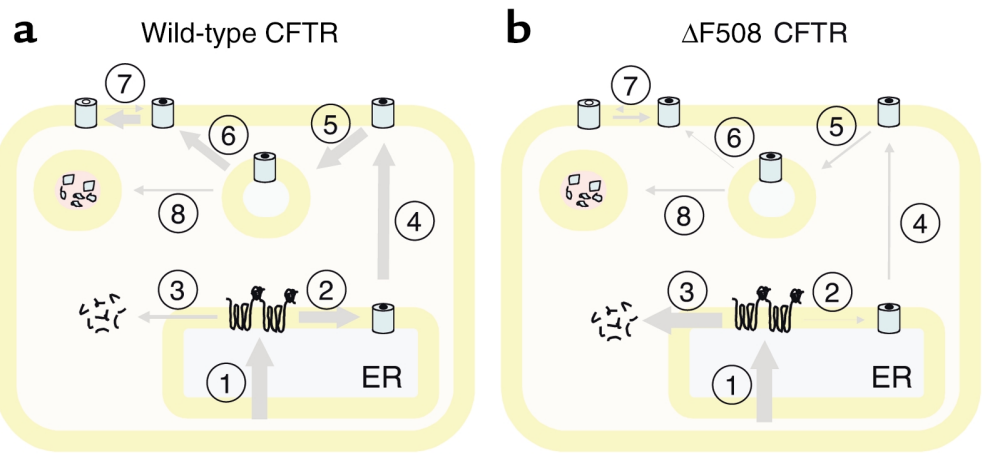

\begin{abstract}
Figure 2
Biogenesis and intracellular trafficking pathway of wild-type (a) and $\Delta$ F508 (b) CFTR. The width of the gray arrows is proportional to the relative flux through a particular branch of the pathway. Synthesis and cotranslational integration (step 1) in the ER membrane are followed by folding to a native conformation (step 2). About $25 \%$ of wild-type and more than $99 \%$ of $\Delta$ F508 CFTR molecules are degraded by a process that is mediated by cytoplasmic proteasomes (step 3). Native CFTR molecules (light blue cylinder) are delivered via the Golgi apparatus (not shown) to the plasma membrane (step 4), where they are subject to rapid endocytosis (step 5) to subapical vesicles (light blue lumen). CFTR is recycled to the plasma membrane (step 6), where it can be activated by cAMP-dependent kinases (step 7). Differences in the relative rates of recycling and degradation in lysosomes (pink lumen; step 8) are likely to account for the substantial differences in half-lives between wild-type and $\Delta$ F508 CFTR.
\end{abstract}

\section{What's wrong with $\triangle \mathrm{F} 508$ CFTR?}

When expressed heterologously in cultured epithelial or nonepithelial cells, $\triangle$ F508 CFTR is found as an immature, core-glycosylated species localized by immunofluorescence microscopy to the ER membrane, whereas wild-type CFTR is predominantly found as a complex glycosylated species at the plasma membrane (4). CFTR immunoreactivity is restricted to internal membranes in sweat ducts from $\triangle \mathrm{F} 508 \mathrm{CFTR}$ homozygotes (7), although recent studies suggest that the degree to which $\triangle$ F508 CFTR is detectable at the plasma membrane may be tissue-specific (8). These findings led to the initial assignment of $\triangle F 508$ CFTR as a class II, or trafficking, mutant (2).

Further study of the function and fate of this mutant protein indicates that the trafficking model is incomplete. As discussed below, folding defects in $\Delta \mathrm{F} 508$ CFTR biosynthesis alter the protein's interactions with the quality control system in the early secretory pathway and also directly or indirectly affect its activity as an anion channel and its stability as a cell surface glycoprotein. Thus, $\Delta \mathrm{F} 508$ should be regarded not as a simple class II mutant, but as a mixed mutant with the properties of classes II, III, and IV.

\section{$\triangle F 508$ CFTR is folding-defective}

Quality control mechanisms in the ER recognize misfolded or partially assembled membrane and secretory proteins and block their deployment to distal compartments, either by retaining them in this compartment through prolonged association with molecular chaperones or by targeting them for degradation (9). Pulse-chase analysis shows that nascent $\Delta$ F508 CFTR molecules are rapidly degraded by the ubiquitin-proteasome system without detectable lag following their release from the ribosome (4). Together with biochemical studies of the thermal stability and refolding properties of recombinant $\Delta$ F508 CFTR fragments, these studies have led to the conclusion that $\Delta$ F508 CFTR is fundamentally a folding mutant (10) and that its steady-state distribution in the ER reflects the operation of the quality control machinery in that organelle, rather than a trafficking defect per se (4). Simply increasing $\Delta \mathrm{F} 508$ CFTR expression in heterologous cells $(11,12)$ leads to increased expression of functional $\Delta$ F508 CFTR molecules at the plasma membrane, indicating that the phenotype of this mutation is leaky. Although this leakiness could be a simple consequence of overwhelming the quality control machinery, it is more likely that deletion of $\mathrm{Phe}^{508}$ reduces the efficiency with which CFTR folds, possibly due to kinetic partitioning of an intermediate in the early folding pathway (Figure 2, step 2). This view of $\Delta \mathrm{F} 508$ CFTR as a folding mutant is also supported by the observation, discussed below, that the fractional folding of $\triangle F 508$ CFTR in vivo (4) (or of a fragment of the protein in vitro [ref. 13]) can be increased by reducing the temperature of incubation or by adding chemical chaperones.

\section{$\Delta$ F508 CFTR gating shows diminished response to $\beta$-adrenergic agonists}

Comprehensive biophysical analysis from many laboratories has established that, while the basic pore properties of $\triangle F 508$ CFTR are indistinguishable from those of wild-type CFTR, the kinetics of CFTR gating is significantly altered by this mutation $(12,14-16)$. The observed difference in macroscopic halide conductance properties can be explained by a three- to fourfold reduction in the channel's open probability, mainly due to increased closed times $(12,15)$. Whether this difference is a direct consequence of a primary structural change in CFTR resulting from deletion of $\mathrm{Phe}^{508}$ or represents a secondary effect of altered interaction with kinases or phosphatases that control channel activation (17), it is clear that $\Delta$ F508 CFTR channel function in situ is severely reduced at any given agonist concentration (Figure 2, step 7).

\section{Rescued $\Delta$ F508 CFTR is less stable than wild-type CFTR}

Once delivered to the plasma membrane (Figure 2, step 4), CFTR is subject to endocytic recycling through a subapical vesicular compartment (5). The steady-state distribution of $\triangle \mathrm{F} 508 \mathrm{CFTR}$ at the plasma membrane is determined by the relative rates of endocytosis (step 5) and exocytosis (step 6). The overall half-life of CFTR molecules in this recycling pool is determined by parti- 
tioning between exocytosis (step 6) and degradation by the lysosome (step 8). Estimates of the half-life of the wild-type plasma membrane/recycling pool of CFTR range from 18-48 hours in BHK cells (18-20) to 24-48 hours in the epithelial line LCPK1. In marked contrast, the half-life of $\triangle$ F508 CFTR is only about 4 hours, as determined in cultured cells exposed to chemical chaperones or held at a reduced temperature (treatments that allow the protein to accumulate on the surface prior to the assay) (18-20). Pulse-chase labeling studies indicate that this reduced half-life is a consequence of increased lysosomal degradation and not simply a redistribution between surface and subapical recycling compartments. The finding that complex glycosylated $\Delta$ F508 CFTR molecules are 20 times less stable than wild-type CFTR at modestly elevated temperatures $\left(40^{\circ} \mathrm{C}\right)$ but exhibit stability similar to that of wild-type CFTR at reduced temperatures suggests that the shorter half-life of plasma membrane $\Delta$ F508 CFTR is due to structural differences between $\Delta \mathrm{F} 508$ and wild-type CFTR (19). Data from proteolytic mapping studies confirm this view.

\section{Pharmacological rescue of $\Delta \mathrm{F} 508$ CFTR trafficking} Because both the instability of the rescued $\Delta$ F508 CFTR at the plasma membrane and the protein's inefficient conformational maturation in the ER are phenotypes of $\mathrm{Phe}^{508}$ deletion, it is possible that this mutant protein is prone to adopt a structurally similar, non-native conformation in both membranes. If so, this conformation may be detected by quality control machinery linked in the ER to ubiquitin-dependent proteolysis but linked in the distal secretory pathway to lysosomal degradation. This model would suggest that a small-molecule drug that facilitates $\Delta$ F508 CFTR folding in the ER might also be effective at stabilizing the protein in the plasma membrane. On the other hand, it is also possible that compounds that specifically suppress off-pathway conformers in the ER do not interact with the non-native conformation in the plasma membrane. In either case, these studies clearly underscore the need to consider $\Delta$ F508 CFTR stability at the plasma membrane in any pharmacological rescue therapy regimen.

Fortunately, there are promising drug candidates capable of increasing CFTR activation kinetics (see ref. 21 for a comprehensive recent review). These compounds, which include the alkylxanthines, flavones, and phosphatase inhibitors, appear to stimulate CFTR by a combination of amplifying the cAMP signal transduction/phosphorylation cascade and directly binding to CFTR. Below, we consider a subset of these compounds that have been reported to ameliorate the $\triangle$ F508 CFTR defect by increasing its delivery to or its stability in the plasma membrane.

\section{Chemical and pharmacological chaperones}

The term "chemical chaperone" loosely describes a family of low-molecular weight compounds including polyols (e.g., glycerol, sorbitol, and myo-inositol), amines (e.g., betaine and trimethylamine- $\mathrm{N}$-oxide [TMAO]), and solvents such as DMSO and $\mathrm{D}_{2} \mathrm{O}$. These compounds have long been recognized to have protein-stabilizing properties in vitro, due largely to their ability to increase protein hydration (22). Endogenously produced compounds like myo-inositol and betaine serve as osmolytes, balancing osmotic forces in cells and organisms that are chronically exposed to osmotic stress.

Incubation of $\Delta$ F508 CFTR-expressing cells in high concentrations $(>1 \mathrm{M})$ of glycerol or other chemical chaperones (e.g., TMAO or $\mathrm{D}_{2} \mathrm{O}$ ) increases the steadystate level of complex-glycosylated $\triangle F 508$ CFTR on immunoblotting and increases functional cAMP-activated channel activity, as seen in measurements of halide efflux and whole-cell patch clamp analysis (23). Pulse-chase analysis shows that the increase in steadystate expression of functional cell surface $\Delta \mathrm{F} 508$ CFTR in the presence of glycerol is due to increased maturation of core-glycosylated nascent $\Delta F 508$ CFTR to a post-ER compartment and decreased degradation, most likely as a result of enhanced folding (24). Fischer and colleagues report significant increases in forskolin-activated intestinal $\mathrm{Cl}^{-}$transepithelial transport in matched wild-type and $\triangle$ F508 CFTR (but not in CFTR-null) mice injected subcutaneously with TMAO, suggesting that a similar rescue of $\Delta \mathrm{F} 508$ CFTR is feasible in vivo (25). This functional restoration was dependent upon the dose of TMAO; at a level near the $\mathrm{LD}_{50}$ for the compound, this treatment supported $\mathrm{Cl}^{-}$transport levels less than one-sixth of those observed in mice expressing wild-type CFTR. These data, together with the demonstration that TMAO and glycerol increase folding of $\triangle F 508$ CFTR in cell culture, confirm the feasibility of pharmacological rescue of at least some misfolded CFTR mutants and should provide the impetus for drug-discovery screens for compounds that are active in rescuing $\Delta \mathrm{F} 508$ CFTR folding at nontoxic pharmacological doses.

A related approach to treating CFTR misfolding involves the use of "pharmacological chaperones" (26), defined substrates or ligands of cell surface-borne channels and receptors. Mutant membrane proteins that have been studied in this regard include P-glycoprotein and the $\mathrm{V} 2$ vasopressin and $\delta$-opioid receptors. The identification of pharmacological chaperones constitutes proof-of-principle demonstration that small, high-affinity compounds can influence the conformational maturation pathways of proteins and ameliorate their folding defects.

\section{Upregulation of CFTR surface expression using butyrate}

Butyrate is a short-chain fatty acid that has long been recognized to function at millimolar concentrations as a transcriptional activator, most likely due to its ability to influence chromatin structure via inhibition of histone deacetylase activity (27). The molecular targets of butyrate have not been identified. Possibly as a direct result of its effects on transcription, or in combination with other mechanisms, butyrate has been reported to modulate a wide spectrum of cellular events, including cellular differentiation and apoptosis (28). 
Treatment of cells expressing $\triangle \mathrm{F} 508$ CFTR under control of the Metallothionein (29) and cytomegalovirus (M. Gelman and R. Kopito, unpublished observations) promoters with sodium butyrate stimulates expression of $\triangle \mathrm{F} 508 \mathrm{CFTR}$, with a concomitant increase in functional CFTR at the cell surface. Elevated levels of $\Delta$ F508 CFTR mRNA and protein synthesis following butyrate treatment suggest that the increase in functional $\triangle \mathrm{F} 508 \mathrm{CFTR}$ is a consequence of overexpression, rather than a specific effect on folding, since no effect of butyrate on folding efficiency or degradation kinetics has been reported. The $\Delta$ F508 CFTR folding defect is leaky, a finding that is best explained by a kinetic model in which a fixed fraction of $\triangle$ F508 CFTR molecules are able to fold and, hence, to mature to the plasma membrane. Wild-type CFTR, which is also folded inefficiently, should likewise be upregulated by this treatment (4). An increase in surface $\Delta$ F508 CFTR can thus be achieved simply by increasing $\Delta$ F508 CFTR gene expression, independent of any effect on trafficking. Whether butyrate and the butyrate analog 4-phenyl butyrate (4PB) influence $\Delta \mathrm{F} 508 \mathrm{CFTR}$ folding or trafficking in addition to their effects on transcription is controversial, as is their efficacy as therapeutic agents. Rubenstein and colleagues tested the effects of $4 \mathrm{~PB}$ on $\Delta$ F508 CFTR expression in primary nasal polyp cultures from CF patients with $\Delta$ F508 CFTR alleles or the immortalized CF cell line 1B3-1 (30). They observed increased forskolin-activated $\mathrm{Cl}^{-}$conductance, concomitant with increased accumulation of the complex glycosylated form of CFTR, and they argued that 4PB alters the intracellular trafficking of $\Delta$ F508 CFTR. However, since they did not report in that study whether butyrate treatment affected $\Delta$ F508 CFTR transcription, and since data on $\triangle F 508$ CFTR folding efficiency and turnover are lacking, the observed increase in functional surface $\Delta$ F508 CFTR might result from enhanced transcription, rather than increased trafficking efficiency. Indeed, Heda and Marino (31) have found that butyrate treatment increased both the amount of functional cell surface $\Delta$ F508 CFTR and $\triangle$ F508 CFTR mRNA levels.

It has been argued that the effect of $4 \mathrm{~PB}$ on $\Delta \mathrm{F} 508$ CFTR trafficking is explained by decreased levels of heat shock cognate protein Hsc70 (32). While this model is not implausible, it remains to be shown whether Hsc70 is limiting for $\Delta$ F508 CFTR degradation. In light of the pleiotropic effects of butyrate on cell function (28), the contradictory data on the effect of butyrate on the inducible heat shock protein $\mathrm{Hsp} 70(32,33)$, and the possibility that butyrate's effect on $\triangle \mathrm{F} 508$ CFTR is simply a consequence of overexpression, it would be premature to conclude that butyrate or $4 \mathrm{~PB}$ influences the folding or the stability of $\triangle$ F508 CFTR.

The utility of butyrates as potential therapeutics may be limited by their inhibitory effects on ion transport systems. Butyrates inhibit CFTR directly $(34,35)$ and may block CFTR function indirectly by inhibiting sodium pumps (36) or other unidentified transporters in the basolateral membrane (37), as well as Na-K-2Cl cotransporters (38), all of which are required for transepithelial salt transport. Given these concerns about the mechanisms and potential side effects of these drugs, additional caution is warranted before proceeding with further clinical trials.

\section{CPX}

The adenosine $\mathrm{A} 1$ receptor 8-cyclopentenyl-1,3-dipropylxanthine (CPX), an alkylxanthine now in phase 2 clinical trials for $\mathrm{CF}$, has been reported to enhance $\mathrm{Cl}^{-}$efflux from NIH3T3 cells expressing $\triangle$ F508 CFTR (39). CPX is also reported to correct the trafficking defect of $\Delta \mathrm{F} 508$ CFTR $(40,41)$. The supporting data consist of immunoblot analyses, immunofluorescence microscopy, and laser scanning cytometry, which together show that CPX treatment leads to increased expression of total $\Delta \mathrm{F} 508$ CFTR and a small concomitant increase in the steadystate levels of a band corresponding to the complex glycoform of CFTR (41). While these data are consistent with CPX improving folding or delivery of $\triangle F 508$ CFTR to the plasma membrane, they are readily explained by an increase in synthesis of this leaky mutant. In the absence of any data directly measuring the effect of the drug on the efficiency of $\Delta$ F508 CFTR folding or exit from the ER, the claim that CPX can repair or correct the trafficking of $\triangle \mathrm{F} 508$ CFTR is unjustified.

\section{Drugs that interfere with molecular chaperones and proteasomal degradation}

The simple model presented in Figure 2 predicts that inhibition of $\triangle F 508$ CFTR degradation should, by mass action, increase production of folded $\Delta \mathrm{F} 508$ CFTR molecules. However, acute or chronic exposure of $\triangle \mathrm{F} 508 \mathrm{CFTR}$-expressing cells to proteasome inhibitors leads to accumulation of detergent-insoluble, multiubiquitylated $\Delta$ F508 CFTR molecules, with no detectable increase in folded CFTR (4). These findings suggest that the substrate of proteasome action is not in direct equilibrium with intermediates on the folding pathway. These and subsequent studies on the degradation of misfolded $\Delta$ F508 CFTR and other proteins in the secretory pathway (see Kaufman, this Perspective series, ref. 42; see also ref. 4) reveal that degradation is a multistep process (Figure 3 ) that involves recognition (step 3a), ubiquitin conjugation and dislocation across the ER membrane (step $3 \mathrm{~b}$ ), and unfolding and degradation by the proteasome (step $3 \mathrm{c}$ ). Proteasome inhibitors (inhibition of step 3c) cause proteins to aggregate in the cytoplasm (step $3 \mathrm{~d}$ ), interfering with the dislocation process (step $3 \mathrm{~b}$ ) (43). Given the strong aggregation-promoting effect of proteasome inhibitors and the accumulating evidence that protein aggregation is a toxic event linked to the pathogenesis of many neurodegenerative diseases (43), therapeutic strategies that target the proteasome or associated machinery should be approached with extreme caution, all the more so because many of these diseases take decades to manifest, so toxic side effects of proteasome inhibitors may fail to be detected by conventional toxicity assays.

A second class of compounds that have been proposed as having potential utility in rescuing $\Delta \mathrm{F} 508$ 


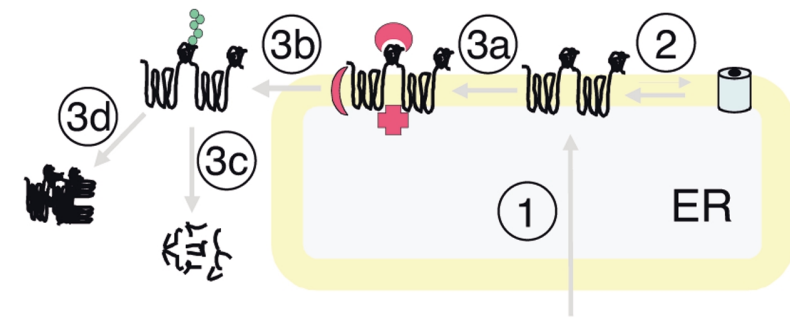

Figure 3

Following initial synthesis and membrane integration (step 1), nascent CFTR molecules are inefficiently folded (step 2) and are recognized as misfolded by one or more components of the quality control machinery (red; step 3a), which may interact with cytoplasmic, membrane, or lumenal portions of CFTR. This interaction is necessary to dislocate CFTR to the cytoplasmic ubiquitin (green spheres) conjugation machinery (step $3 b$ ), which then directs it to the proteasome for degradation (step 3c). Cytoplasmic aggregates form (step 3d) when the rate of production and dislocation of misfolded CFTR exceeds the capacity of the ubiquitin-proteasome system for degradation.

CFTR misfolding are those which target molecular chaperones systems. Nascent CFTR molecules interact transiently with cytoplasmic members of the Hsp70 (44, 45), Hsp40 (45), and Hsp90 (46) families and the ER lumenal chaperone calnexin (47); and these chaperones may contribute to recognition of misfolded CFTR and $\triangle$ F508 CFTR. The immunosuppressant deoxyspergualin (DSG) binds Hsp70 and Hsp90 in vitro with micromolar affinity and appears to compete for binding of substrates to a subset of these chaperones (48). Treatment of $\Delta$ F508 CFTR-expressing epithelial cells with DSG increases forskolin-stimulated halide efflux and whole-cell currents to levels comparable to those achieved by low temperature incubation (49), suggesting that, at least at rather high concentrations $(50 \mu \mathrm{g} / \mathrm{ml})$, this drug can partially restore function to $\Delta$ F508 CFTR. However, there are no data to support the hypothesis that DSG influences the folding or delivery to the plasma membrane, directly or indirectly. Even though it appears to exhibit some specificity with respect to Hsp70 family members (50), the pleiotropic effects of interfering with both the Hsp70 and the Hsp90 chaperone systems could have indirect consequences on multiple cellular pathways, including those that influence CFTR activation or gating mechanisms. Moreover, although it is clear that DSG interacts with Hsp70 and Hsp90, there is no evidence that any of the biological effects of this drug are mediated by the drug's interaction with these chaperones; other targets of lower abundance and higher affinity may remain to be identified. Interestingly, treatment of CFTR-expressing cells with the benzoquinone ansamycins geldanamycin and herbimycin $\mathrm{A}-$ drugs that bind with high affinity to Hsp90-family chaperones - appears to perturb the interaction between Hsp90 and CFTR, suppressing its maturation and accelerating its degradation (46). Recent studies suggest that geldanamycin activates a general heat shock response, influencing other classes of molecular chaperones and components of the degradation apparatus, perhaps as an indirect consequence of its effects on Hsp90 (51).

A recent report by Egan et al. (52) suggests that thapsigargin (TG), an inhibitor of the ER calcium pump, can also increase expression of forskolin-stimulated $\mathrm{Cl}^{-}$ channels and immunochemically detectable $\Delta \mathrm{F} 508$ CFTR molecules in the apical plasma membrane. This drug is proposed to enhance $\Delta$ F508 CFTR egress from the ER by interfering with its interaction with one or more Ca-dependent ER chaperones. Although TG treatment leads to an increase in the complex glycosylated form of $\triangle F 508$ CFTR, the data do not rule out the possibility that this drug directly or indirectly influences the partitioning of $\Delta$ F508 CFTR molecules through the endocytic pathway (Figure 2a, steps 7 and 8). Although the authors show that TG treatment does not appear to induce a general heat shock response, its well-established role as an inducer of the "unfolded protein response" (UPR; see Kaufman, this Perspective series, ref. 42; and ref. 53) suggests that its effect on $\triangle$ F508 CFTR might be nonspecific. It will be important to determine whether other UPR inducers such as tunicamycin have similar effects. Perturbation of cellular Ca homeostasis and ER chaperone function and the attendant impact on signal transduction, protein folding, and degradation may promote proteotoxicity over the long term, despite the reported lack of acute toxicity in $\triangle$ F508 CFTR mice (52).

Molecular chaperones play essential roles both in protein biogenesis, where they facilitate folding and suppress aggregation, and in protein degradation, where they contribute to recognition and destruction of misfolded molecules. The studies described here suggest that drugs that interfere with the action of broad classes of molecular chaperones can influence the functionality of $\triangle$ F508 CFTR molecules in model systems. Concerns over the utility of chaperone antagonists or agonists center on our lack of understanding of the role of specific chaperones in the intracellular fate of $\Delta \mathrm{F} 508$ CFTR, and a recognition of the pleiotropic nature of chaperone action and of the potential for induction of proteotoxicity through aggregate formation.

Clearly, considerable basic investigation of these questions is required before this class of compounds can be considered as potential therapeutics. Perhaps ubiquitin E3 ligases or other relatively specific components of the quality control apparatus will offer more reasonable targets. Because E3s participate in the earliest stages of $\triangle$ F508 CFTR recognition (Figure 3, step 3a), identification of E3s in $\triangle F 508$ CFTR degradation should be viewed as an important goal.

\section{Other compounds reported to influence $\triangle$ F508 CFTR trafficking}

Substituted benzo[c]quinolizinium (MBP) compounds MBP-07 and MBP-91 have also been reported to activate wild-type and $\triangle \mathrm{F} 508 \mathrm{CFTR} \mathrm{Cl}$ conductance in cultured $\triangle$ F508 CFTR epithelial cells $(54,55)$. These compounds appear to act at the low millimolar range to increase CFTR Cl transport function, and limited biochemical data suggest that they can increase the amount of com- 
plex glycosylated $\Delta$ F508 CFTR and immunohistochemically detectable staining at the apical plasma membrane of nasal epithelial cells. Additional data will be needed to rule out the possibility that they simply increase $\Delta$ F508 CFTR transcription or stabilize the plasma membrane pool of $\Delta$ F508 CFTR. Moreover, the observation that these compounds increase intracellular $\triangle$ F508 CFTR staining in a juxtanuclear location (55) suggests that MBP-07 and MBP-91 may actually promote $\Delta$ F508 CFTR aggregation.

The anthracycline doxorubicin (Dox) was recently reported to increase functional cell surface expression of $\triangle \mathrm{F} 508 \mathrm{CFTR}(56)$. Low concentrations $(0.25 \mu \mathrm{M})$ of this drug promote increased halide efflux and shortcircuit current in monolayers of T84 cells and appear to produce a modest increase in steady-state accumulation of complex glycosylated CFTR without apparent effect on CFTR mRNA levels. While these data suggest that Dox could increase the efficiency of CFTR folding or stabilize the plasma membrane pool, the effect observed was quite transient: Enhanced CFTR expression was observed only 24 hours after Dox treatment and was lost by 48 hours. It is also curious that Dox has no significant effect on the activity or processing of CFTR in another epithelial cell line, MDCK. Finally, the extremely modest effect of this compound on the cell surface expression of $\triangle F 508$ CFTR and the absence of evidence indicating functional activation of $\Delta \mathrm{F} 508$ CFTR, together with the considerable toxicity of this anticancer drug, raise doubts about its utility as a drug.

\section{Prospects for pharmacological rescue of $\triangle$ F508 CFTR}

The initial optimism surrounding the discovery that the most common CF allele, $\Delta \mathrm{F} 508$, is mislocalized and functional has been tempered by the finding that the protein is not a simple trafficking mutant but exhibits multiple defects in folding, stability, and activation. Practically, this implies that for successful pharmacological rescue it may be essential to identify drugs that ameliorate all three phenotypes. Because the pathways on which these $\Delta \mathrm{F} 508$ defects lie are distinct (Figure $2 \mathrm{~b}$ ), it is likely that compounds affecting any single pathway will act synergistically. For example, because the cell surface half-life of $\Delta$ F508 CFTR is so significantly reduced, it is likely that any treatment that enhances $\Delta$ F508 CFTR folding will be greatly enhanced by even a modest prolongation of cell surface residence. In support of this view, Heda and Marino report that sodium butyrate, a transcriptional enhancer, acts synergistically with reduced temperature, a treatment that increases folding efficiency (31).

Recognition of the multiple $\Delta$ F508 CFTR phenotypes must be considered in the design of any rational highthroughput screen for pharmacological agents to rescue $\Delta$ F508 CFTR. Screening efforts currently underway are focused on cell-based reporters that can identify compounds that increase cAMP-dependent transmembrane halide movement (57). These assays have the advantage of identifying any compound that increases CFTR function, since they make no assump- tion about mechanism. On the other hand, small effects of any compound on a single pathway could be greatly enhanced if combined with a well-defined effector of another pathway. An alternative approach would be to customize screens to identify compounds that act on a specific phenotype - for example, to search for molecules that increase the amount of cell surface $\triangle$ F508 CFTR irrespective of function. Such a screen could identify molecules that enhance either folding or stability of the mutant protein, and it might yield either potential leads or compounds that could be useful in conjunction with functionally based screens.

In summary, there is reason to expect that pharmacological rescue of $\Delta$ F508 CFTR is an achievable goal. However, the compounds that have been thus far described to ameliorate the folding or stability defect are either unsuitable for pharmacological use because of inherent toxicity (e.g., chemical chaperones like TMAO and glycerol) or too poorly characterized at the cellular or molecular level to support the claims that they specifically rescue or correct the $\Delta \mathrm{F} 508$ trafficking defect (e.g., CPX, 4PB, MBP, and Dox). The optimal strategy in the development of effective therapeutics for CF will be to simultaneously target the defects in folding, stability, and activation of $\triangle$ F508 CFTR. This goal will require a more complete understanding of the mechanism of action of currently available and newly discovered drugs.

\section{Acknowledgments}

The authors are grateful to G. Endemann for critical reading of the manuscript.

1. Kerem, B.-S., et al. 1989. Identification of the cystic fibrosis gene: genetic analysis. Science. 245:1073-1080.

2. Welsh, M.J., and Smith, A.E. 1993. Molecular mechanisms of CFTR chloride channel dysfunction in cystic fibrosis. Cell. 73:1251-1254.

3. Wine, J.J. 1999. The genesis of cystic fibrosis lung disease. J. Clin. Invest. 103:309-312

4. Kopito, R.R. 1999. Biosynthesis and degradation of CFTR. Physiol. Rev. 79(Suppl.):S167-S173.

5. Kleizen, B., Braakman, I., and de Jonge, H.R. 2000. Regulated trafficking of the CFTR chloride channel. Eur. J. Cell Biol. 79:544-556.

6. Riordan, J.R., et al. 1989. Identification of the cystic fibrosis gene: cloning and characterization of complementary DNA. Science. 245:1066-1073.

7. Kartner, N., Augustinas, O., Jensen, T.J., Naismith, A.L., and Riordan, J.R. 1992. Mislocalization of delta F508 CFTR in cystic fibrosis sweat gland. Nat. Genet. 1:321-327.

8. Kälin, N., Claaß, A., Sommer, M., Puchelle, E., and Tümmler, B. 1999. $\triangle$ F508 CFTR protein expression in tissues from patients with cystic fibrosis. J. Clin. Invest. 103:1379-1389.

9. Ellgaard, L., Molinari, M., and Helenius, A. 1999. Setting the standards: quality control in the secretory pathway. Science. 286:1882-1888.

10. Qu, B.H., Strickland, E., and Thomas, P.J. 1997. Cystic fibrosis: a disease of altered protein folding. J. Bioenerg. Biomembr. 29:483-490.

11. Cheng, S.H., et al. 1995. Functional activation of the cystic fibrosis trafficking mutant DF508-CFTR by overexpression. Am. J. Physiol. 268:L615-L624.

12. Dalemans, W., et al. 1991. Altered chloride ion channel kinetics associated with the DF508 cystic fibrosis mutaion. Nature. 354:526-528.

13. Qu, B.H., and Thomas, P.J. 1996. Alteration of the cystic fibrosis transmembrane conductance regulator folding pathway. J. Biol. Chem. 271:7261-7264

14. Drumm, M.L., et al. 1991. Chloride conductance expressed by DF508 and other mutant CFTRs in Xenopus oocytes. Science. 254:1797-1799.

15. Haws, C.M., et al. 1996. Delta F508-CFTR channels: kinetics, activation by forskolin, and potentiation by xanthines. Am. J. Physiol. 270:C1544-C1555.

16. Denning, G.M., et al. 1992. Processing of mutant cystic fibrosis trans- 
membrane conductance regulator is temperature-sensitive. Nature. 358:761-764.

17. Li, C., et al. 1993. The cystic fibrosis mutation (delta F508) does not influence the chloride channel activity of CFTR. Nat. Genet. 3:311-316.

18. Lukacs, G.L., et al. 1993. The delta F508 mutation decreases the stability of cystic fibrosis transmembrane conductance regulator in the plasma membrane. Determination of functional half-lives on transfected cells. J. Biol. Chem. 268:21592-21598.

19. Sharma, M., Benharouga, M., Hu, W., and Lukacs, G.L. 2001. Conformational and temperature-sensitive stability defects of the delta F508 cystic fibrosis transmembrane conductance regulator in post-endoplasmic reticulum compartments. J. Biol. Chem. 276:8942-8950.

20. Heda, G.D., Tanwani, M., and Marino, C.R. 2001. The Delta F508 mutation shortens the biochemical half-life of plasma membrane CFTR in polarized epithelial cells. Am. J. Physiol. Cell Physiol. 280:C166-C174.

21. Roomans, G.M. 2001. Pharmacological treatment of the ion transport defect in cystic fibrosis. Expert Opin. Investig. Drugs. 10:1-19.

22. Gekko, K., and Ito, H. 1990. Competing solvent effects of polyols and guanidine hydrochloride on protein stability. J. Biochem. (Tokyo). 107:572-577.

23. Brown, C.R., Hong-Brown, L.Q., and Welch, W.J. 1997. Strategies for correcting the delta F508 CFTR protein-folding defect. J. Bioenerg. Biomem br. 29:491-502.

24. Sato, S., Ward, C.L., Krouse, M.E., Wine, J.J., and Kopito, R.R. 1996. Glycerol reverses the misfolding phenotype of the most common cystic fibrosis mutation. J. Biol. Chem. 271:635-638.

25. Fischer, H., et al. 2001. Partial restoration of defective chloride conductance in DeltaF508 CF mice by trimethylamine oxide. Am. J. Physiol. Lung Cell. Mol. Physiol. 281:L52-L57.

26. Morello, J.P., Petaja-Repo, U.E., Bichet, D.G., and Bouvier, M. 2000. Pharmacological chaperones: a new twist on receptor folding. Trends Pharmacol. Sci. 21:466-469.

27. Kruh, J. 1982. Effects of sodium butyrate, a new pharmacological agent, on cells in culture. Mol. Cell. Biochem. 42:65-82.

28. Marks, P.A., Richon, V.M., and Rifkind, R.A. 2000. Histone deacetylase inhibitors: inducers of differentiation or apoptosis of transformed cells. J. Natl. Cancer Inst. 92:1210-1216.

29. Cheng, S.H., et al. 1995. Functional activation of the cystic fibrosis trafficking mutant delta F508-CFTR by overexpression. Am. J. Physiol. 268:L615-L624.

30. Rubenstein, R.C., Egan, M.E., and Zeitlin, P.L. 1997. In vitro pharmacologic restoration of CFTR-mediated chloride transport with sodium 4 phenylbutyrate in cystic fibrosis epithelial cells containing delta F508CFTR. J. Clin. Invest. 100:2457-2465.

31. Heda, G.D., and Marino, C.R. 2000. Surface expression of the cystic fibrosis transmembrane conductance regulator mutant DeltaF508 is markedly upregulated by combination treatment with sodium butyrate and low temperature. Biochem. Biophys. Res. Commun. 271:659-664.

32. Rubenstein, R.C., and Zeitlin, P.L. 2000. Sodium 4-phenylbutyrate downregulates Hsc70: implications for intracellular trafficking of DeltaF508CFTR. Am. J. Physiol. Cell Physiol. 278:C259-C267.

33. Choo-Kang, L.R., and Zeitlin, P.L. 2001. Induction of HSP70 promotes DeltaF508 CFTR trafficking. Am. J. Physiol. Lung Cell. Mol. Physiol. 281:L58-L68.

34. Linsdell, P. 2001. Direct block of the cystic fibrosis transmembrane conductance regulator $\mathrm{Cl}(-)$ channel by butyrate and phenylbutyrate. Eur. J. Pharmacol. 411:255-260.

35. Loffing, J., Moyer, B.D., Reynolds, D., and Stanton, B.A. 1999. PBA increases CFTR expression but at high doses inhibits $\mathrm{Cl}(-)$ secretion in Calu-3 airway epithelial cells. Am. J. Physiol. 277:L700-L708.

36. Moyer, B.D., Loffing-Cueni, D., Loffing, J., Reynolds, D., and Stanton, B.A. 1999. Butyrate increases apical membrane CFTR but reduces chloride secretion in MDCK cells. Am. J. Physiol. 277:F271-F276.

37. Loffing-Cueni, D., et al. 2001. Trafficking of GFP-tagged DeltaF508CFTR to the plasma membrane in a polarized epithelial cell line. Am.J.
Physiol. Cell Physiol. 281:C1889-C1897.

38. Matthews, J.B., et al. 1998. Na-K-2Cl cotransporter gene expression and function during enterocyte differentiation. Modulation of $\mathrm{Cl}^{-}$secretory capacity by butyrate. J. Clin. Invest. 101:2072-2079.

39. Guay-Broder, C., et al. 1995. A1 receptor antagonist 8-cyclopentyl-1,3dipropylxanthine selectively activates chloride efflux from human epithelial and mouse fibroblast cell lines expressing the cystic fibrosis transmembrane regulator delta F508 mutation. Biochemistry. 34:9079-9087.

40. Srivastava, M., Eidelman, O., and Pollard, H.B. 1999. Pharmacogenomics of the cystic fibrosis transmembrane conductance regulator (CFTR) and the cystic fibrosis drug CPX using genome microarray analysis. Mol. Med. 5:753-767.

41. Eidelman, O., Zhang, J., Srivastava, M., and Pollard, H.B. 2001. Cystic fibrosis and the use of pharmacogenomics to determine surrogate endpoints for drug discovery. Am. J. Pharmacogenomics. 1:221-238.

42. Kaufman, R.J. 2002. Orchestrating the unfolded protein response in health and disease. J. Clin. Invest. 110:1389-1398. doi:10.1172/ JCI200216886.

43. Kopito, R.R. 2000. Aggresomes, inclusion bodies and protein aggregation. Trends Cell Biol. 10:524-530.

44. Yang, Y., Janich, S., Cohn, J.A., and Wilson, J.M. 1993. The common variant of cystic fibrosis transmembrane conductance regulator is recognized by hsp70 and degraded in a pre-Golgi nonlysosomal compartment. Proc. Natl. Acad. Sci. USA. 90:9480-9484.

45. Meacham, G.C., et al. 1999. The Hdj-2/Hsc70 chaperone pair facilitates early steps in CFTR biogenesis. EMBO J. 18:1492-1505.

46. Loo, M.A., et al. 1998. Perturbation of Hsp90 interaction with nascent CFTR prevents its maturation and accelerates its degradation by the proteasome. EMBO J. 17:6879-6887.

47. Pind, S., Riordan, J.R., and Williams, D.B. 1994. Participation of the endoplasmic reticulum chaperone calnexin (p88, IP90) in the biogenesis of the cystic fibrosis transmembrane conductance regulator. J. Biol. Chem. 269:12784-12788.

48. Nadler, S.G., Tepper, M.A., Schacter, B., and Mazzucco, C.E. 1992. Interaction of the immunosuppressant deoxyspergualin with a member of the Hsp70 family of heat shock proteins. Science. 258:484-486.

49. Jiang, C., et al. 1998. Partial restoration of cAMP-stimulated CFTR chloride channel activity in DeltaF508 cells by deoxyspergualin. Am. J. Physiol. 275:C171-C178.

50. Brodsky, J.L. 1999. Selectivity of the molecular chaperone-specific immunosuppressive agent 15-deoxyspergualin: modulation of Hsc70 ATPase activity without compromising DnaJ chaperone interactions. Biochem. Pharmacol. 57:877-880.

51. Sittler, A., et al. 2001. Geldanamycin activates a heat shock response and inhibits huntingtin aggregation in a cell culture model of Huntington's disease. Hum. Mol. Genet. 10:1307-1315.

52. Egan, M.E., et al. 2002. Calcium-pump inhibitors induce functional surface expression of DeltaF508-CFTR protein in cystic fibrosis epithelial cells. Nat. Med. 8:485-492.

53. Bertolotti, A., Zhang, Y., Hendershot, L.M., Harding, H.P., and Ron, D. 2000. Dynamic interaction of BiP and ER stress transducers in the unfolded-protein response. Nat. Cell Biol. 2:326-332.

54. McPherson, M.A., et al. 2001. The CFTR-mediated protein secretion defect: pharmacological correction. Pflugers Arch. 443(Suppl. 1):S121-S126.

55. Dormer, R.L., et al. 2001. Correction of delF508-CFTR activity with benzo(c)quinolizinium compounds through facilitation of its processing in cystic fibrosis airway cells. J. Cell Sci. 114:4073-4081.

56. Maitra, R., Shaw, C.M., Stanton, B.A., and Hamilton, J.W. 2001. Increased functional cell surface expression of CFTR and DeltaF508-CFTR by the anthracycline doxorubicin. Am. J. Physiol. Cell Physiol. 280:C1031-C1037.

57. Galietta, L.V., Jayaraman, S., and Verkman, A.S. 2001. Cell-based assay for high-throughput quantitative screening of CFTR chloride transport agonists. Am. J. Physiol. Cell Physiol. 281:C1734-C1742. 